To recreate bone's nanostructure in the laboratory, Stupp and his team designed a cone-shaped molecule, a peptide-amphiphile, that is bulkier and hydrophilic on one end (a peptide) and slimmer and hydrophobic on the other (an alkyl group). When in water at low $\mathrm{pH}$, the molecules assemble themselves like spokes on a wheel, with the hydrophobic greasy tail directed to the center, leaving the peptide to face the exterior aqueous environment. This basic structure is repeated so that a long nanofiber is formed, similar to an insulated copper wire where the insulation is the peptide and the wire is the alkyl group. The synthetic fibers orient the growth of the hydroxyapatite crystals so that they mimic the structure found in natural bone.

The researchers engineered their peptide structure to attract bone cells, but the chemistry of the peptide is customizable, said Stupp, and can be changed to attract different cells to the fibrous scaffold, such as neurons, cartilage, muscle, liver, and pancreas cells.

Stupp said, "We've mimicked this for bone, but we have offered a strategy that would work for other tissues of the human body, or to create materials inspired by bone that could be useful in electronics or photonics."

\section{Electrical Conductivity of Single-Molecule Wires Accurately Measured}

Researchers from Arizona State University have determined a method for creating through-bond electrical contacts with single molecules, enabling them to achieve reproducible measurements of the molecules' conductivity. ASU chemistry professor Devens Gust said, "Progress in the field has been hampered by two problems. The first has been in making robust, reproducible electrical connections to both ends of molecules. After this has been achieved, the next problem is knowing how many molecules there actually are between the electrical contacts."

As reported in the October 19, 2001, issue of Science, the researchers began with a uniform atomic layer of gold atoms and attached octanethiol insulator molecules to it through chemical bonds, forming a coating of aligned molecules. Using a solvent, they removed some of the insulators and replaced them with molecules of 1,8-octanedithiol, a molecule that is similar but capable of bonding with gold at both ends and acting as a molecular "wire."

Gold 2-nm particles were then added to the solvent, where they bonded to the free ends of the 1,8-octanedithiol mole- cules, thus creating a bonded metallic contact at either end of the conducting molecules. A gold-coated conducting atomic force microscope probe was then run across the surface and conductivity was measured when it made contact with the gold particles.

When electrical measurements were made on over 4000 gold particles, virtually all measurements fell into one of five groups of distinct conductivity curves, according to the researchers. The conductivity curves were distinct whole-number multiples of a single, fundamental curve, they said.

The researchers said that the fundamental curve represents conduction by a single molecule of octanedithiol attached to the two gold contacts. When more than a single molecule was bound, each additional molecule increased the current capacity by the single unit amount of current that could be carried by one molecule. When the probe encountered octanethiol insulator molecules, which could not bond with a gold particle, a much higher electrical resistance was recorded.

Gust said, "The molecule becomes a much better conductor when it is 'soldered' into the circuit by the bonds to gold at each end. This suggests how we can wire single-molecule components into a molecular circuit board, and lays some important groundwork for doing practical molecular electronics."

\section{Cubic ZnMgO Alloys Offer Alternative to III-Nitride Wurtzite Structure}

Jagdish Narayan and his associates at North Carolina State University have synthesized $\mathrm{ZnMgO}$ alloys that have a cubic sodium chloride structure, which may provide an alternative to III-nitrides of wurtzite hexagonal structure. When $\mathrm{ZnO}$ is alloyed with $\mathrm{MgO}$, it can be controlled to produce either a hexagonal (wurtzite) or cubic (sodium-chloride) structure, they said. The cubic structure can be grown epitaxially on $\mathrm{Si}(100)$ by domain-matching epitaxy, where four lattice constants of the silicon match with three of the substrate. In addition, $\mathrm{ZnO}$ and its alloys have a higher exciton binding energy (60 meV) as compared with III-nitride counterparts ( $25 \mathrm{meV})$, which results in less trapping of carriers and luminescent efficiencies, they said.

$\mathrm{ZnO}$, a II-VI semiconductor with a bandgap of $3.27 \mathrm{eV}$ at $300 \mathrm{~K}$, compares very closely with $\mathrm{GaN}$ of III-V systems. To vary the bandgap of $\mathrm{ZnO}$, it can be alloyed with $\mathrm{MgO}(8.2 \mathrm{eV})$ to increase the bandgap or with $\mathrm{CdO}(2.0 \mathrm{eV})$ to decrease it. This is similar to the III-nitride system,
Narayan said, where GaN is alloyed with AlN (6.2 eV) to increase the bandgap or with AlN to decrease it. These III-nitride alloys have a hexagonal (wurtzite) structure, which can be grown epitaxially only on substrates of hexagonal symmetry. These substrates include $\alpha-\mathrm{Al}_{2} \mathrm{O}_{3}$, (III)Si, $6 \mathrm{H}-\mathrm{SiC}$, and $\mathrm{ZnO}$. The epitaxial growth on $6 \mathrm{H}-\mathrm{SiC}$ and $\mathrm{ZnO}(0001)$ substrates occurs by lattice-matching epitaxy due to a small misfit. On the other hand, Narayan said, the epitaxial growth of III-nitride on $\alpha-\mathrm{Al}_{2} \mathrm{O}_{3}$ and (111)Si occurs by means of domain-matching epitaxy, where an integral multiple of major planes/lattice constants of the film match with the substrate. For example, six (0110) planes of the film match with the seven (1210) planes of the substrate after a $30^{\circ} / 90^{\circ}$ rotation in the basal plane, he said.

The $\mathrm{ZnMgO}$ alloys can be used to produce any color, including the three primary colors needed for white light-emitting devices that would be more durable and consume less power than conventional incandescent bulbs or fluorescent lamps, Narayan said.

"The shorter wavelength can also be focused more tightly, leading to a substantial increase in storage capacity of magnetic and optical disks," he said.

The researchers anticipate further applications for their U.S. patented alloy in the field of spintronics, biosensors, and optical communications.

\section{Nanosphere Converted to Nanoprism, Offering Different Properties}

Scientists at Northwestern University have been able to create triangular nanoprisms. They reported the method used to produce triangular nanoprisms in large quantities, using commercially available silver nanospheres, soap, and visible light, in the November 30, 2001, issue of Science.

In nanotechnology, most of the bulk preparatory methods are for spheres and rods, said Chad A. Mirkin, director of Northwestern's Institute for Nanotechnology, who led the experimental portion of the study.

In their experiments, the researchers found that when they placed common nanospheres made of silver in a solution containing soap molecules and irradiated the mixture with room light for three days, the spheres were converted into triangular prisms $15 \mathrm{~nm}$ thick.

The light induced the nanospheres to break up into silver atoms, which then fed the growth of the nanoprisms, a process called ripening. The conversion process could be arrested at any point by 
stopping the light exposure, thus giving the researchers control over the shape and size of the nanoparticles.

"When you go from a sphere to a prism you get new properties-new optical properties, electrical properties, chemical properties, and catalytic properties," said Mirkin, who is the George B. Rathmann Professor of Chemistry at Northwestern. "It's the same material, but a different shape and size. And that makes all the difference."

\section{Polythiophene Nanowires Written onto Si Wafers}

Chemists at Duke University have prepared polythiophene nanowires on semiconducting and insulating Si wafers using an atomic force microscope-based direct-writing technique: electrochemical dip-pen nanolithography. Faculty members J. Liu and M.W. Grinstaff and graduate students B.W. Maynor and S. Filocamo report in the January 30 issue of the Journal of the American Chemical Society that 3,4-ethylenedioxythiophene (EDOT) can be electrochemically polymerized at an AFM tip/Si surface interface to afford well-defined sub-100-nm wires of polyEDOT. To pattern these nanostructures, an EDOT-coated AFM tip is translated across the $\mathrm{Si}$ wafer while a negative bias voltage is applied to electrochemically polymerize the monomer. Nanowire morphology is controlled by applied voltage and tip translation speed.

According to Grinstaff and Liu, the capability to pattern poly-EDOT on insulating surfaces provides a means to use this polymer in future nanodevices. When combined with other micro- and nanofabrication techniques, this approach provides opportunities for designing and developing devices for a wide range of applications in the electronics, pharmaceutical, and biotechnological industries, they said.

\section{Supercritical-Fluid-Extraction Method Cleans Radioactive- Contaminated Soil}

By applying a ligand-assisted supercriticalfluid-extraction method, chemists at the Idaho National Engineering and Environmental Laboratory have decontaminated a sample of soil spiked with ${ }^{239} \mathrm{Pu}$ and ${ }^{241} \mathrm{Am}$. They used pressurized, heated carbon dioxide and an added metal-binding chemical compound to clean the soil, removing $>69 \%$ of the plutonium and americium.

As reported in the October 2001 issue of Radiochemica Acta, a chemical agent added to the carbon dioxide flowed through the soil and grabbed the plutonium and americium, whisking the compound back into the fluid-like carbon dioxide. The carbon dioxide was then shunted out of the soil and depressurized, dropping the compound into a vial on its way back into the atmosphere. The researchers added ethanol and said they could add different chemical agents to improve the efficiency of the extraction.

The chemists reported that the supercritical-fluid-extraction method leaves the soil intact. Chemist Robert Fox said that other methods used, such as nitric-acid extraction, dissolved $25 \%$ of the soil mass. He said, "Dissolving soil in nitric acid creates a radioactive sludge that must still be disposed of."

The chemists said that the effectiveness of the supercritical-fluid-extraction method in removing radioactive elements from the soil depends partly on the chemistry of the soil. Soil particles are made up of minerals from rocks and clay, which react differently with radioactive elements. They said plutonium that is bound near the surface of a particle is easier to remove than that bound inside the mineral lattice.

In follow-up experiments, the researchers were able to remove close to $100 \%$ of the contamination. While they confirmed that their spiked soil serves as a suitable surrogate for a real-world sample, the chemists acknowledge that weather and aging can affect the chemistry of the materials bound to the soil.

Fox said, "We also want to have a fundamental understanding of the chemistry that occurs-why does it work that way, and what is inhibiting it from working faster and better."

\section{Nanoscale Computing Machine Built with DNA Molecules}

A group of scientists headed by Ehud Shapiro of the Computer Science and Applied Mathematics Department and the Biological Chemistry Department at the Weizmann Institute of Science, Israel, has used DNA molecules to create a nanocomputer. As reported in the November 22, 2001, issue of Nature, Shapiro and graduate student Yaakov Benenson used a solution consisting of DNA molecules and two naturally occurring DNA-manipulating enzymes: Fok-I and ligase. Fok-I functions as a chemical scissors, cleaving DNA in a specific pattern, whereas the ligase enzyme seals DNA molecules together.
The research team used the four DNA bases $A, G, C$, and $T$ to encode the input data as well as the program rules underlying the computer "software." Both input and software molecules were designed to have one DNA strand longer than the other, resulting in a single-strand overhang called a "sticky end."

Two molecules with complementary sticky ends can temporarily stick to each other-known as hybridization-allowing DNA ligase to permanently seal them into one molecule. The sticky end of the input molecule encodes the current symbol and the current state of the computation, whereas the sticky end of each "software" molecule is designed to detect a particular state-symbol combination. A two-state, two-symbol automaton has four such combinations. For each combination, the nanocomputer has two possible next moves, to remain in the same state or to change to the other state, allowing eight software molecules to cover all possibilities.

The researchers reported that in each processing step, the input molecule was hybridized with a software molecule that had a complementary sticky end, allowing ligase to seal them together using two ATP molecules as energy. Then the Fok-I detected the recognition site in the software molecule.

It cleaved the input molecule in a location determined by the software molecule, thus exposing a sticky end that encoded the next input symbol and the next state of the computation. Once the last input symbol was processed, a sticky end encoding the final state of the computation was exposed and detected, again by hybridization and ligation, by one of two "output display" molecules. The resulting molecule, which reported the output of the computation, was made visible to the human eye by gel electrophoresis.

While the nanocomputer is too simple to have immediate applications, the researchers said it may pave the way for future computers that can operate within the human body with biological and pharmaceutical applications.

\section{FOR MORE RESEARCH NEWS ON MATERIALS SCIENCE ... \\ ... access the Materials Research Society Web site: \\ www.mrs.org/gateway/ matl_news.html}

